\title{
PREDICTIVE TOOLS IN PROSTATE CANCER
}

\author{
Anakievski D., Al. Hinev \\ Clinic of Urology, Department of Surgery, St. Marina University Hospital of Varna
}

\begin{abstract}
Accurate assessment of the pathological tumour stage and treatment outcomes is of utmost importance for the patient with prostate cancer and for his treating physician. Nomograms are graphical statistical tools that use different variables to calculate the potential risk. They provide estimates based on the specific characteristics of the individual patient. They are objective prognostic tools that should be taken into consideration when making treatment decisions. A brief review summarizing the most popular prognostic tables and nomograms most commonly used in prostate cancer is presented. The advantages and disadvantages of these prognostic instruments, the predictive accuracy of each one of them, as well as some results from the external validation studies are discussed in detail.
\end{abstract}

Key words: prostate cancer, prognosis, survival, nomograms, validation

\section{INTRODUCTION}

In most industrialized countries, cancer of the prostate gland (PCa) is the most common malignancy among men and third leading cause of death in them. The introduction of prostate-specific antigen (PSA) screening for men aged over 50 years and the new improvements in biopsy techniques led to a real 'boom' in PCa diagnosis. Early diagnosis of the disease in turn increases the chance of men as a definitive treatment and better quality of life after its implementation.

$\mathrm{PCa}$ is a heterogeneous disease with a very wide range of forecasting. Patients face with a variety of treatment options such as radical prostatectomy (RP), telegamatherapy, hormonoterapy, cryotherapy, HIFU and active surveillance, each of them to different extent, resulting in a significant change of quality of life.

Over the past decade, RP has become a treatment method of choice in cases of localized PCa (13). The main goals of surgery are the following: complete eradication of malignant disease, i.e. absence of recurrence and tumour progression (cancer control) to maximize the life expectancy (increasing cancer-specific and overall survival) and restitution quality of life primarily to control sexual and urinary function.

Accurate assessment of the likelihood for success of treatment and possible complications that can significantly affect quality of life and lead to long-term morbidity are essential for the patient when making the right decision concerning treatment choice. In patients with proven $\mathrm{PCa}$, ac-

\footnotetext{
Address for correspondence:

D. Anakievski, Clinic of Urology, Dept. of Surgery, Medical University of Varna, 55 Marin Drinov Str., Varna 9002, Bulgaria e-mail: dejan_anakievski@yahoo.com
}

curate prediction of pathologic stage determining the risk of presence of disease in an advanced stage or risk of occurrence of relapse after radical treatment applied is very important. In them, we have considered additional information which is disease-specific such as age, patient's comorbidities, life expectancy, potential toxicity of the treatment administered, etc.

There is, therefore, a pressing need for tools to assist patients and their physicians in clinical analysis enabling the correct decision. Such instruments are prognostic nomograms which have gained popularity in the last decade.

Nomograms are graphical statistical tools that use different variables to calculate the potential risk. They provide individual estimates based on single patient's characteristics derived from hundreds or even thousands of patients with the same disease.

The ideal nomogram should be accurate, not influenced by cultural and socio-economic differences as well as not dependent on the pathologist who made the pathomorphogical assessment or the surgeon who did the operation. It should be generally applicable to any patients rather than to those operated in elite institutions using strict selection criteria only.

The clinical value of each nomogram is determined mainly by the prognostic accuracy of the calculated correlation between estimated prediction and actual risk. It takes into consideration whether such prediction is achieved using an external data set different from that used in the creation of nomogram.

Based on the used variables nomograms are divided into preoperative and postoperative. Preoperative nomograms can be used to decide which treatment will be best for the patient according to the pathological state and its individual prognostic profile. On the other hand, the precise risk estimation is important for patients who already have under- 
gone RP. Early prediction of relapse of the disease helps doctors identifying the patients at high risk, modulating the postoperative surveillance and optimizing their treatment. However, it should be emphasized that the nomogram gives predicted result only rather than definitive information about symptomatic disease progression or treatment-related complications.

The first introduction of a nomogram in medicine is in 1928 by Lawrence Joseph Henderson who is a biochemist by profession. He describes in more than 100 graphics the interaction of various factors affecting the blood-gas transport and physiology of blood as a physico-chemical system.

Many nomograms for a variety of diseases, including for PCa have been created and introduced into clinical practice (16).

The first prognostic tool in $\mathrm{PCa}$ consists in a prognostic table for assessing the pathological stage using a simple combination of clinical parameters such as preoperative serum PSA, biopsy Gleason score and clinical stage (13). Significant change in the attitude of clinicians to the practical use of nomograms occurs after the publication of nomogram to assess the outcome after definitive local therapy for PCa based on several clinical before treatment variables (11). A nomogram for predicting the presence of $\mathrm{PCa}$ in biopsy cylinders from the initial biopsy in men who had suspicious physical findings on digital rectal examination and normal serum levels of PSA $(<4,0 \mathrm{ng} / \mathrm{mL})$ was first developed (6). The use of sextant biopsies significantly restricted the applicability of this nomogram.

A similar nomogram (3) was developed for the patients undergoing extended biopsy. It included percentage of free PSA as an additional variable and was externally validated in 2900 male patients divided into three groups demonstrating an accuracy of $76 \%$.

Newly developed nomograms prove that the combination of clinical variables such as preoperative serum PSA, biopsy Gleason score and clinical stage much better predict pathological stage than any of these variables considered separately. Based on the information about these three variables, Partin's tables calculate the probability of the presence of organ confined disease (OCD), extraprostatic extension (EPE), invasion of seminal vesicles (SVI) and of lymph nodes (LNI). Although Partin's tables represent a milestone in PCa pre-staging, they have their limitations. Despite criticism they find wide application and are validated not only in USA, but also in other countries in Europe and Asia.

Models to predict the presence of EPE are developed, too (8) allowing for identifying candidates for nerve-sparing (unilateral or bilateral) RP with an accuracy of $70 \%$ according to the external validation. A nomogram with predictive probability of EPE by the side of the prostate based on some preoperative variables such as $\mathrm{cT}$, Gleason score, percentage of positive cylinders, percentage of Tu into the cylinders on each side, and PSA has been developed presenting with an accuracy of $84 \%$ (12). Based on clinical stage, biopsy Gleason score and PSA value, three groups of patients with different risk to EPE have been identified (5):
- low risk - sT1c-sT2a, Gleason score $<7$ and PSA $<10 \mathrm{ng} / \mathrm{mL}$;

- moderate risk - sT2b, Gleason score of 7 and PSA of $10-20 \mathrm{ng} / \mathrm{mL}$, and

- high risk - sT2c or more, Gleason score of 8-10 and PSA $>20 \mathrm{ng} / \mathrm{mL}$.

Other nomograms predict predict the likelihood of SVI in patients diagnosed with extended biopsy (10 or more biopsy cylinders) by using PSA, biopsy Gleason score, cT and percentage of positive cylinders with an accuracy of $78 \%-88 \%$ (7). Another nomogram predicts LNI with a higher accuracy as compared to Partin's tables when tested in the same group of patients with the same clinical variables (2). A similar nomogram designed for $\mathrm{LNI}$ in patients with clinically localized PCa (1). This nomogram uses PSA values, clinical stage, biopsy Gleason score and number of dissected lymph nodes by ELND. In this case, there is a linearly increased risk of LNI in the patients undergoing RP proportionally to the number of dissected lymph nodes. The accuracy of the nomogram itself is of $80 \%$ and allows for accurate identification of the need of extended pelvic lymphadenectomy. Nowadays, some nomograms $(5,10,11)$ are most commonly appled to predict the likelihood of biochemical relapse before and after RP.

Han's tables (10) are created in the same institution like Partin's ones and are based on information about three basic prognostic variables such as PSA value, biopsy Gleason score and clinical stage in the preoperative model, while in postoperative model the results of biopsy Gleason score is replaced by a surgical result and the clinical stage by the pathological stage determined after surgery. The preoperative and postoperative models calculate the probability of occurrence of biochemical relapse on the 3rd, 5th, 7th and 10th year following operation.

D'Amico's nomogram (5) provides prognostic information about the occurrence of biochemical relapse in patients treated with RP and radiation therapy as well as in patients with a high density of positive biopsies following endorectal MRI usage. The main criticism of this nomogram concerns the definition used for biochemical recurrence, i.e. three consecutive PSA elevationsin in surgically treated patients.

Kattan's nomograms (11) along with preoperative and postoperative nomograms of Memorial Sloan-Kettering Cancer Center (MSKCC) in New York are among the most popular and widely used worldwide. They assess the risk of recurrence based on clinical variables such as serum PSA, clinical stage and biopsy Gleason score and inform about the slowly growing $\mathrm{PCa}$. Postoperative nomogram provides more accurate prognostic information compared with preoperative one due to the additional pathologic information obtained after prostatectomy. Unlike other nomograms, there is opportunity to introduce additional, more detailed prognostic information, such as prostate volume, number of previous biopsy procedures with negative results, information about the total tumour length in positive biopsy cylinders, information about total length of benign prostatic tissue in biopsy cylin- 
ders, the percentage of tumour tissue compared to benign prostatic biopsy cylinders from the base of the prostate and the average frequency of metastases in the lymph nodes in patients treated by LND and the RP in their own institution. Based on this information, final result nomogram determines probability (in percentage) of presence - OCD, EPE, SVI, and LNI as well as the probability of the disease to progress after RP (in the 5th and 10th year after surgery, respectively) and the cancer-specific survival rate (in the $10^{\text {th }}$ and $15^{\text {th }}$ year following surgery, respectively).

Four nomograms have been developed for predicting the survival rate of PCa patients. Porter's nomogram (14) predicts cancer-specific survival rate of PCa patients undergoing hormone therapy, regardless of time of its initiation. The remaining 3 nomograms predict the likelihood of survival in the patients with androgen-insensitive $\mathrm{PCa}$ $(9,17,18)$.

Life expectancy plays a crucial role in treatment, especially in patients who are candidates for RP. A 10-year survival rate is, usually, considered minimal life expectancy of men with PCa. Unfortunately, the first nomograms for predicting the life expectancy were not sufficiently reliable in the potential candidates for RP as their accuracy did not exceed $60 \%$. Later on, nomograms were developed to predict life expectancy in men with RP $(4,19)$. The accuracy of these models ranged from $69 \%$ to $73 \%$. A nomogram was developed for predicting the life expectancy exceeding 10 years in the candidates for RP (20). This nomogram focused on the patients with relapses after definitive treatment. Internal validation of the nomogram demonstrated an accuracy of $84 \%$.

The nomograms should be externally validated and tested in real practice. The original model should be applied to a new data set that should originate from an external source in order to assess the performance of a nomogram. Regression coefficients taken from the original model are applied to each patient individually either to derive probabilities (if logistic regression is used for this original), or to determine linearly the prediction (if regression model of Cox is used). The predictive accuracy of the nomogram is determined by the area under the ROC curve for the analysis - the greater that area, the higher the predictive accuracy of the nomogram.

Informamtion retrieval of nomograms for $\mathrm{PCa}$ in MEDLINE database from 1966 to 2010 identifies 109 published nomograms that can be administered to the patients in different clinical stages of disease. Of them, only 68 nomograms have already undergone validation (15).

\section{REFERENCES}

1. Briganti, A., F. K. Chun, A. Salonia, A. Gallina, E. Farina, L. F. Da Pozzo, et al. Validation of a nomogram predicting the probability of lymph node invasion based on the extent of pelvic lymphadenectomy in patients with clinically localized prostate cancer.- BJU. Int., 98, 2006, No 4, 788-793.

2. Cagiannos, I., P. Karakiewicz, J. A. Eastham, M. Ohori, F. Rabbani, C. Gerigk, et al. A preoperative nomogram identifying decreased risk of positive pelvic lymph nodes in patients with prostate cancer.- J. Urol., 170, 2003, No 5, 1798-1803.

3. Chun, F. K., P. I. Karakiewicz, A. Briganti, J. Walz, M. W. Kattan, H. Huland, et al. A critical appraisal of logistic regression-based nomograms, artificial neural networks, classification and regression-tree models, look-up tables and risk-group stratification models for prostate cancer.- BJU. Int., 99, 2007, No 4, 794-800.

4. Cowen, M. E., L. K. Halasyamani, M. W. Kattan. Predicting life expectancy in men with clinically localized prostate cancer.- J. Urol., 175, 2006, No 1, 99-103.

5. D'Amico, A. V., Y. Wu, M. H. Chen, M. Nash, A. A. Renshaw, J. P. Richie. Perineural invasion as a predictor of biochemical outcome following radical prostatectomy for select men with clinically localized prostate cancer.- J. Urol., 165, 2001, No 1, 126-129.

6. Eastham, J. A., R. May, J. L. Robertson, O. Sartor, M. W. Kattan. Development of a nomogram that predicts the probability of a positive prostate biopsy in men with an abnormal digital rectal examination and a prostate-specific antigen between 0 and 4 ng/mL.- Urology, 54, 1999, No 4, 709-713.

7. Gallina, A., F. K. Chun, A. Briganti, S. F. Shariat, F. Montorsi, A. Salonia, et al. Development and split-sample validation of a nomogram predicting the probability of seminal vesicle invasion at radical prostatectomy.- Eur. Urol., 52, 2007, No 1, 98-105.

8. Graefen, M. Kombination von prostataspezifischem Antigen, klinischem Stadium und Gleason-Grad zur Vorhersage des pathologischen Stadiums des lokalisierten Prostatakarzinoms - eine multiinstitutionale Erneuerung.- Aktuelle Urol., 35, 2004, No 5, 377-378.

9. Halabi, S., E. J. Small, P. W. Kantoff, M. W. Kattan, E. B. Kaplan, N. A. Dawson, et al. Prognostic model for predicting survival in men with hormone-refractory metastatic prostate cancer.J. Clin. Oncol., 21, 2003, No 7, 1232-1237.

10. Han, M., A. W. Partin, M. Zahurak, S. Piantadosi, J. I. Epstein, P. C. Walsh, et al. Biochemical (prostate specific antigen) recurrence probability following radical prostatectomy for clinically localized prostate cancer.- J. Urol., 169, 2003, No 2, 517-523.

11. Kattan, M. W., J. A. Eastham, A. M. Stapleton, T. M. Wheeler, P. T. Scardino. A preoperative nomogram for disease recurrence following radical prostatectomy for prostate cancer.- $J$. Natl. Cancer Inst., 90, 1998, No 10, 766-771.

12. Ohori, M., M. W. Kattan, H. Koh, N. Maru, K. M. Slawin, S. Shariat, et al. Predicting the presence and side of extracapsular extension: a nomogram for staging prostate cancer.- J. Urol., 171, 2004, No 5, 1844-1849; discussion, p. 1849.

13. Partin, A. W., J. Yoo, H. B. Carter, J. D. Pearson, D. W. Chan, J. I. Epstein, et al. The use of prostate specific antigen, clinical stage and Gleason score to predict pathological stage in men 
with localized prostate cancer.- J. Urol., 150, 1993, No 1, 110-114.

14. Porter, C. R., A. Galliana, K. Kodama, R. P. Gibbons, R. Correa, Jr, P. Perrotte, et al. Prostate cancer specific survival in men treated with hormonal therapy after failure of radical prostatectomy.- Eur. Urol., 52, 2007, No 2, 446-452.

15. Shariat, S. F., P. I. Karakiewicz, C. G. Roehrborn, M. W. Kattan. An updated catalog of prostate cancer predictive tools.- Cancer, 113, 2008, No 11, 3075-3099.

16. Shariat, S. F., J. Walz, C. G. Roehrborn, F. Montorsi, C. Jeldres, F. Saad, et al. Early postoperative plasma transforming growth factor-beta1 is a strong predictor of biochemical progression after radical prostatectomy.- J. Urol., 179, 2008, No 4, 1593-1597.

17. Smaletz, O., H. I. Scher, E. J. Sma1l, D. A. Verbel, A. McMillan, K. Regan, et al. Nomogram for overall survival of patients with pro- gressive metastatic prostate cancer after castration.- $J$. Clin. Oncol., 20, 2002, No 19, 3972-3982.

18. Svatek, R. S., C. Jeldres, P. I. Karakiewicz, N. Suardi, J. Walz, C. G. Roehrborn, et al. Pre-treatment biomarker levels improve the accuracy of post-prostatectomy nomogram for prediction of biochemical recurrence.Prostate, 69, 2009, No 8, 886-894.

19. Tewari, A., C. C. Johnson, G. Divine, E. D. Crawford, E. J. Gamito, R. Demers, et al. Long-term survival probability in men with clinically localized prostate cancer: a case-control, propensity modeling study stratified by race, age, treatment and comorbidities.- J. Urol., 171, 2004, No 4, 1513-1519.

20. Walz, J., A. Gallina, F. Saad, F. Montorsi, P. Perrotte, S. F. Shariat, et al. A nomogram predicting 10-year life expectancy in candidates for radical prostatectomy or radiotherapy for prostate cancer.- J. Clin. Oncol., 25, 2007, No 24, 3576-3581. 